\title{
EFFECTS OF IN-VEHICLE MESSAGING ON MENTAL WORKLOAD DURING DRIVNG THROUGH WORK ZONES
}

Curtis M. Craig, Jacob Achtemeier, Brady Patzer, and Nichole L. Morris HumanFIRST Laboratory, Dept. of Mechanical Engineering, University of Minnesota Minneapolis, MN, USA

Email: craigc@umn.edu,jachteme@umn.edu,bpatzer@umn.edu,nlmorris@umn.edu

\begin{abstract}
Summary: Driving through work zones is especially risky. This study investigates a potential strategy for warning drivers of important events during work zone driving using in-vehicle smartphone messages. Participants used a driving simulator to drive through two different work zones. Work zone events were communicated either by roadside signage, through audio messages or through audio-visual messages on a smartphone placed either on the dashboard or the passenger seat of the vehicle. Subjective measures of mental workload and usability, along with event recall, were recorded for each drive. The overall pattern of results suggests reduced mental workload and better usability for in-vehicle smartphone messages. In-vehicle message systems may be a promising approach for improving driver safety in the work zone.
\end{abstract}

\section{INTRODUCTION}

\section{Work Zones}

Driving is the riskiest activity in which people ordinarily engage, and driving through work zones is especially dangerous given the low error margin. The work zone environment is volatile, often requiring abrupt lane changes and braking. Drivers may not maintain adequate situation awareness to notice these traffic changes in a work zone, leading to heightened risk of crashes (Wang, Hughes, Council, \& Paniati, 1996). Typical work zone signs communicating risks or speed limits are not very effective in reducing speeds (Fontaine, Schrock, \& Ullman, 2002). Variable message and speed limit signs are more effective at promoting speed reduction (Bertini, Boice, \& Bogenberger, 2006). However, drivers in work zones may miss critical information altogether due to distraction or inattention (Muttart, Fisher, Knodler, \& Pollatsek, 2007).

Distraction provided by smartphones and other mobile devices have a well-known contribution to poor driving performance (Strayer \& Johnson, 2001). However, smartphones are capable of presenting task-relevant messages in relation to the context of driving, and task-relevant messages are less disruptive than task-irrelevant messages (Czerwinski, Cutrell, \& Horvitz, 2000). The focus of the present study was to determine whether in-vehicle smartphone messages about work zone hazards could promote better attention to the message content, while maintaining or reducing the mental workload of the driving task.

\section{Mental Workload and Driving}

Recarte and Nunes (2003) demonstrated that driving under conditions of higher mental demand leads to increased spatial gaze concentration and fewer speedometer and rearview mirrors 
checks. Also, higher mental workload during stimulated driving resulted in reduced detection and discrimination of critical targets, implying a risk of reduced hazard perception during high task demand conditions (Recarte \& Nunes, 2003). For this reason, any implementation of invehicle messaging via smartphones must not substantially increase the mental demand imposed by the driving task.

Furthermore, because conditions of elevated mental workload imposed by the driving route itself may shape gaze patterns, the placement of the smartphone in the visual field may have important human factors implications. A model of visual scanning, SEEV, predicts that people will be less likely to move their visual attention to areas that are distant from current focus due to the mental effort required (Wickens, 2014). The mental resources required could be scarce under challenging driving environments, such as work zones, reducing the likelihood of attending to in-vehicle messages that are distant from the central visual focus (ideally the road).

\section{Location/Placement}

Drivers report being more likely to place their smartphones in the passenger seat or cup-holders rather than the dashboard, the latter being closer to the road view (Achtemeier \& Morris, 2016). Therefore, not only does the presence of in-vehicle messaging need to be assessed in terms of mental workload, the physical location needs to be considered, as well. Mental workload may be alleviated by the presence of in-vehicle messages, allowing drivers to transfer some of the visual task demand of actively scanning for work zone hazards to the smartphone and allocate freed attentional resources to the primary driving task (Jamson \& Merat, 2005).

\section{Modality}

A third consideration for in-vehicle messages is modality. Presenting purely visual messages may not be effective for capturing attention in the driving environment with the primary task being dominantly visual and visual-motor. Parkes and Coleman (1990) had participants perform a computerized navigation task with different route guidance modalities: A central visual cue, a peripheral visual cue, or an auditory cue. They found that the auditory condition had the fastest performance, the lowest reported workload, and the most gaze time on the primary visual navigation task. Therefore, auditory-only smartphone messages are a possible design consideration. However, audio-visual smartphone messages could be both attention-capturing and easily understood due to redundant coding, leading to both comprehension and minimal impact on mental workload (Wickens, Hollands, Banbury, \& Parasuraman, 2013).

\section{The Present Study}

We consider the impact of in-vehicle messaging in work zones on mental workload, while also considering system usability and user preference. If mental workload is not affected or even reduced by the presence of in-vehicle messages, this should be reflected in both subjective mental workload reporting and recall of the work zone relevant messages. This prediction was tested in a driving simulator, with participants driving two work zone routes. Participants experienced portable changeable message signage (PCMS) on the side of the road representing a 
control condition, an in-vehicle smartphone that had a visual and auditory component, and an invehicle smartphone that had only an auditory component. The placement of the smartphone varied between participants, with a passenger seat smartphone placement condition and a dashboard mounted smartphone placement condition.

\section{METHOD}

Participants. There were 48 participants (23 females, 25 males), with an average age of 25.63 years $(S D=6.02)$. All participants reported some driving experience and cell phone use. All participants had normal or corrected-to-normal vision and no color-blindness.

Apparatus and materials. Participants performed the driving session using a partial motion-based driving simulator manufactured by Realtime Technologies, Inc., consisting of a Saturn SC2 full vehicle cab featuring realistic control operation and instrumentation, including power assist for the brakes and force feedback for the steering. The simulated rural driving route was projected to a five-channel, 210-degree forward visual field screen (2.5 arc-minutes per pixel) with rear and side mirror views provided by a rear screen and vehicle-mounted LCD panels.

Participants completed a driving history survey, the Rating Scale Mental Effort (RSME) questionnaire (Zijlstra, 1993), and the System Usability Scale (SUS; Brooke, 1986). The driving history survey evaluated participant driving history, demographic information, and items related to willingness to engage in cell phone use (e.g. texting, calling) and navigation (e.g. 511, Google Maps) usage while driving. Additionally, a 23-question component of the survey gauged safety culture outlook regarding work zones and roadside information delivery, including items that addressed attitudes on conventional signing, interest and acceptance of using surrogate signing information means (e.g. in-vehicle technologies), and safety perception of drivers in the fleet (Achtemeier \& Morris, 2016).

Alert messages within the vehicle were presented through a 7-inch LCD display. The display presented an image of a smartphone at scale. The visual component presented black text on an orange caution background, and a graphical icon to indicate the nature of the work zone event. Audio alerts consisted of three short spoken phrases for the event context, distance, and suggested driver action. The audio used a computerized female voice at a measured intensity of 82-78 dB (depending on display location). Ambient noise intensity at $55 \mathrm{mph}$ was measured at approximately $65 \mathrm{~dB}$, resulting in a signal-to-noise ratio of +17 to $+13 \mathrm{~dB}$.

Design. The design was a $3 \times 2 \times 2$ mixed factorial with message modality (Control, Audio Only, Audio-Visual), and routes (Northbound, Southbound) as within-subjects measures and smartphone placement (Dash Mounted, Passenger Seat) as a between-subjects measure. Participants were randomly assigned to a smartphone placement group and the order of message modality was counterbalanced across participants using a balanced Latin Square. The secondary task cognitive load was consistent across participants.

Tasks. Each driving route was based on a four-lane median-separated highway design spanning $14.8 \mathrm{~km}$ and modeled a Minnesota rural highway composed of mostly straightaways and a few 
gentle curves. The two driving routes differed in work zone design, as the northbound route featured a shoulder closure with two lanes of travel, whereas the southbound route included a lane closure with one lane of travel. The roadside geography of the simulated route was typical of rural Midwestern highways, featuring rolling plains and wooded regions that provided a consistent clear sight distance and field of view for the participants. The work zone components of the routes followed the MUTCD (MUTCD, 2009) standards for active work zones. The simulated routes were designed to maximize the exposure of relevant, practical uses for the system within the context of an active work zone, ensuring ecological validity and saliency. The northbound shoulder closure included typical work zone events: slow traffic, debris in a lane of travel, trucks entering the roadway, roadside heavy machinery, and a crash blocking a lane of travel. Likewise, the southbound lane closure route incorporated realistic work zone events, including an active work area, gravel in the lane of travel, workers on site, and stopped traffic to simulate bottleneck queuing.

Participants completed a secondary task concurrent with the driving task, designed to induce cognitive load. This was a self-paced backwards counting task in which participants were asked to count backwards by 3 . The beginning number was randomly chosen from 100 to 999 .

Procedure. After providing written consent, participants were tested with a standard Snellen Acuity Chart and Ishihara's color test to ensure they had normal vision. Participants then completed the demographic, driving history, and cell phone use questionnaire. After being fitted for eye-tracking measurement (data not presented here), the participants completed a brief practice run in the simulator to acclimate to the simulator. Participants also practiced the secondary backwards counting task. The practice duration was that of half a total drive, three to five minutes long, and concluded when the participants reported comfort driving the simulator and reported fully understanding the countback task. Participants drove through the two work zone routes (Northbound, Southbound) counterbalanced for each within-subjects condition of message modality (Control, Audio Only, Audio-Visual). Each drive took approximately 10 minutes to complete. During each drive, participants were exposed to five work zone messages (e.g., stopped traffic, debris on road). After each modality was completed, participants completed the RSME and SUS questionnaires, followed by a prompted free recall exercise that assessed their recall of the work zone messages. After the driving task, participants were asked which of the three message modalities they preferred. Afterwards, the participants were debriefed.

\section{RESULTS}

Mental workload. A $3 \times 2 \times 2$ mixed factorial ANOVA was conducted with RSME score as the dependent variable and higher numbers reflecting higher workload. There was a main effect of modality, Greenhouse-Geisser corrected, $F(1.664,74.74 .883)=12.691, p<.001, \eta^{2}=.217$. Post-hoc follow up analyses found the RSME score for the Control condition $(M=52.596, S E=$ 2.880) was significantly greater than the Audio Only condition $(M=43.351, S E=2.990), t(46)$ $=4.008, p<.001$, and the Audio-Visual condition $(M=41.904, S E=3.110), t(46)=4.647, p<$ .001 . There was no difference between the Audio Only and Audio-Visual conditions, $t(46)=$ $.638, p=.409$. See Figure 1. There were no other significant main effects or interactions. 


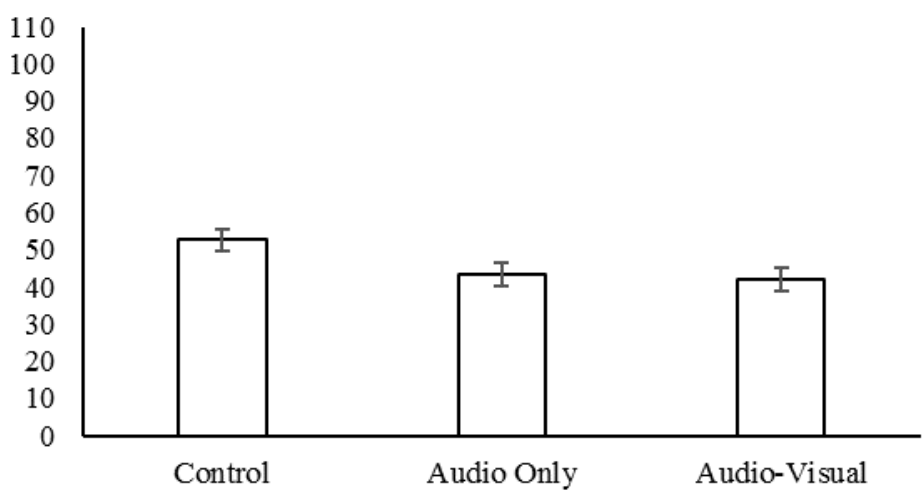

Figure 1. RSME scores measuring mental workload for each modality

Recall. A $3 \times 2 \times 2$ mixed factorial ANOVA was conducted with event recall proportion correct scores as the dependent variable. There was a main effect of modality, $F(1.635,73.597)=$ 26.733, $p<.001, \eta^{2}=.367$. Post-hoc follow up analyses found the recall score for the Control condition $(M=.407, S E=.035)$ was significantly lower than the scores for the Audio Only condition $(M=.509, S E=.033), t(46)=2.856, p=.007$. There was no significant difference between the Control condition and the Audio-Visual condition $(M=.474, S E=.035), t(46)=$ $1.883, p=.087$, and no significant difference between the Audio Only and Audio-Visual conditions, $t(46)=.972, p=.289$. There was also a significant three-way modality $\times$ route $\times$ placement interaction, $F(2,88)=4.035, p=.021, \eta^{2}=.080$. Breaking the three-way interaction down by placement, there was a simple interaction for the passenger seat placement condition for modality $\times$ route, $F(2,44)=4.001, p=.024, \eta^{2}=.150$. Breaking this interaction down further by route, only the southbound (lane closure) route had an effect of modality, $F(2,46)=$ $6.365, p=.004, \eta^{2}=.217$. The recall score for the Control condition $(M=.348, S E=.067)$ was significantly less than the Audio Only condition $(M=.530, S E=.056), t(22)=2.970, p=.007$, and the Audio-Visual condition $(M=.522, S E=.057), t(22)=2.600, p=.016$. There was no difference between the Audio Only and Audio-Visual conditions, $t(22)=.165, p=.870$.

Usability. A $3 \times 2 \times 2$ mixed factorial ANOVA was conducted with System Usability Scale (SUS) scores as the dependent variable. There was a main effect of modality, GreenhouseGeisser corrected, $F(1.635,73.597)=26.733, p<.001, \eta^{2}=.367$. Post-hoc follow up analyses found the SUS score for the Control condition $(M=73.162, S E=2.314)$ was significantly less than the Audio Only condition $(M=86.284, S E=1.782), t(46)=6.344, p<.001$, and the Audio-Visual condition $(M=86.234, S E=1.721), t(46)=6.320, p<.001$. There was no difference between the Audio Only and Audio-Visual conditions, $t(46)=.024, p=.974$.

Preference. A chi-square test of goodness of fit was used to verify whether the three modalities were equivalently preferred. The preference was not equally distributed, $X^{2}(2, N=48)=46.5, p$ $<.001$. The Audio Only modality was preferred by 38 participants, 2 participants preferred the outside signage (Control) modality, and 8 participants preferred the Audio-Visual modality. 


\section{DISCUSSION}

The present study tested whether in-vehicle messaging could contribute to work zone awareness without increasing mental workload, by exposing participants in a driving simulator to multiple work zone events via multiple modalities and two different areas of phone placement. The audio only and audio-visual messaging conditions demonstrated reduced mental workload and better usability scores than the roadside PCMS control condition. There was also a significant main effect of modality for recall, with more events remembered for the audio only messaging condition relative to the control. Finally, for the southbound route (involving lane closure work zone operations), recall scores for the audio only and audio-visual condition was better than the PCMS control condition when the smartphone was placed in the passenger seat.

The primary concern of using in-vehicle messaging during driving was the risk of distraction and increased workload (Strayer \& Johnson, 2001). The presented results imply that, relative to the present design of roadside PCMS work zone signs, in-vehicle message systems with smartphones lead to reduced mental workload and better usability. This highlights a potential intervention to reduce accident risk near and within work zones. Furthermore, recall of the work zone events was better for the in-vehicle smartphone conditions, especially for the audio only design. This implies more efficient attentional allocation to messages without visual content to compete with the visual driving task (Wickens, 2008).

Given that drivers often place their smartphones on locations other than the dashboard (Achtemeier \& Morris, 2016), it is pertinent to observe that recall was higher for work zone events in the in-vehicle message conditions relative to control when driving the southbound route, when the phones were on the passenger seat. The southbound route had participants drive through a lane closure, restricting them to a single lane during the work zone activity area, while the northbound route involved shoulder work and did not require participants to merge into a single lane. The southbound route may have been more stressful, leading participants to limit allocation of their visual attention away from the roadside signage and other extraneous visual information. Therefore, smartphone placement may be less of a concern than previously thought when it comes to the design and deployment of in-vehicle messaging systems.

Finally, although workload and usability scores were similar across the audio-visual and audio only modalities, most of the participants preferred the audio only design. Participants may be sensitive to the danger of visual distraction while driving and prefer the mode that least presents this distraction. Future research should consider the relative advantages of these two modalities and along with other potential uses of driving relevant in-vehicle messaging.

\section{ACKNOWLEDGEMENTS}

This work was funded by the Minnesota Department of Transportation (MnDOT) through sponsor award number 99008, work order 184. We extend special thanks to all the HumanFIRST researchers who contributed to the project, and in particular to Peter Easterlund, Disi Tian, Pooja Bujjuri, and Nick Knudsen. 


\section{REFERENCES}

Achtemeier, J. D., \& Morris, N. L. (2016). An assessment of safety culture while navigating work zones attitudes and behavior toward in-vehicle messaging technologies. In Proceedings of the Human Factors and Ergonomics Society Annual Meeting (Vol. 60, No. 1, pp. 1499-1503). SAGE Publications.

Bertini, R., Boice, S., \& Bogenberger, K. (2006). Dynamics of variable speed limit system surrounding bottleneck on german autobahn. Transportation Research Record, (1978), 149-159.

Czerwinski, M., Cutrell, E. \& Horvitz, E. (2000). Instant messaging: Effects of relevance and time. In S. Turner \& P. Turner (Eds.), People and Computers XIV: Proceedings of HCI 2000, Vol. 2, British Computer Society, pp. 71-76.

Fontaine, M., Schrock, S., \& Ullman, G. (2002). Feasibility of real-time remote speed enforcement for work zones. Transportation Research Record, (1818), 25-31.

Jamson, A. H., \& Merat, N. (2005). Surrogate in-vehicle information systems and driver behaviour: Effects of visual and cognitive load in simulated rural driving. Transportation Research Part F: Traffic Psychology and Behaviour, 8(2), 79-96.

Manual on Uniform Traffic Control Devices. Federal Highway Administration (MUTCD). (2009). U.S. Department of Transportation, Washington, D.C., 2009.

Muttart, J. W., Fisher, D. L., Knodler, M., \& Pollatsek, A. (2007). Driving simulator evaluation of driver performance during hands-free cell phone operation in work zones: Driving without a clue. Transportation Research Record: Journal of the Transportation Research Board, (2018), 9-14.

Parkes, A.M. \& Coleman, N. (1990). Route guidance systems: A comparison of methods of presenting directional information in the driver. In E J Lovesey (Ed.) Contemporary Ergonomics 1990, (pp. 480-485). Taylor \& Francis: London, United Kingdom.

Recarte, M. A., \& Nunes, L. M. (2003). Mental workload while driving: Effects on visual search, discrimination, and decision making. Journal of Experimental Psychology: Applied, 9(2), 119137.

Strayer, D. L., \& Johnston, W. A. (2001). Driven to distraction: Dual-task studies of simulated driving and conversing on a cellular telephone. Psychological Science, 12(6), 462-466.

Wang, J., Hughes, W., Council, F., \& Paniati, J. (1996). Investigation of highway work zone crashes: What we know and what we don't know. Transportation Research Record, (1529), 54-62.

Wickens, C. D. (2014). Effort in human factors performance and decision making. Human Factors, 56(8), 1329-1336.

Wickens, C. D. (2008). Multiple resources and mental workload. Human Factors, 50(3), 449-455.

Wickens, C. D., Hollands, J. G., Banbury, S., \& Parasuraman, R. (2013). Engineering psychology \& human performance. Pearson.

Zijlstra, F.R.H. (1993). Efficiency in work behavior. A design approach for modern tools. $\mathrm{PhD}$ thesis, Delft University of Technology. Delft, The Netherlands: Delft University Press. 